\title{
The Design of Finite-State Machines for Quantization Using Simulated Annealing
}

\author{
Ercan Engin Kuruoğ/u \\ Electrical and Electronics Engineering Department \\ Bilkent University \\ Ankara, 06533, Turkey
}

\author{
Ender Ayanoğlu \\ AT\&T Bell Laboratories \\ 101 Crawfords Corner Road 4F-507 \\ Holmdel, NJ 07733-3030, USA
}

\section{ABSTRACT}

In this paper, the combinatorial optimization algorithm known as simulated annealing is used for the optimization of the trellis structure or the next-state map of the decoder finite-state machine in trellis waveform coding. The generalized Lloyd algorithm which finds the optimum codebook is incorporated into simulated annealing. Comparison of simulation results with previous work in the literature shows that this combined method yields coding systems with good performance.

\section{Introduction}

A high-performance waveform coding technique is known as trellis, lookahead, or delayed decision source or waveform coding [1]. Trellis waveform coding uses a finite-state machine as the decoder. This machine is defined by an output map, corresponding to the codebook, and a next-state map, corresponding to the trellis structure, both of which being functions of the channel symbol and the current state. The extension of the next-state map or the state transition diagram in time is known as a trellis structure, a weighted directed graph consisting of identical stages. Each stage corresponds to a time instant. The encoder is matched to the decoder, it examines the treilis and finds the channel sequence that leads to minimum distortion, which is the sum of the distortion values between the input and reproduction symbols. This can be accomplished by a trellis search algorithm, such as the Viterbi Algorithm (VA). The encoder in a trellis waveform coding system is simply a trellis search algorithm matched to the decoder finite-state machine. Therefore, the design problem reduces to the design of the decoder finite-state machine. This problem has been addressed by several authors in the literature, see, e.g. [1], [2]. The design of the finite-state machine for a quantizer, using a trellis search, or in the context of finite-state vector quantization, without any search, has also been addressed in the literature, see, e.g., [3]. In this work, we optimize both the codewords and the finite-state machine structure of a scalar trellis waveform coder that uses the Viterbi algorithm, using a near-optimum approach. For the optimization of the decoder finite-state machine, we make the observation that since the decoder is equivalent to the trellis structure, for a given set of codewords, and a given input sequence, it is clear that finding the optimum decoder is equivalent to finding the trellis structure that will generate a channel sequence with minimum distortion at the decoder output. This is a combinatorial optimization problem and can be solved by known optimization methods. In this paper, we propose the simulated annealing algorithm [4] for this purpose.

\section{The Design Method}

In this work, the state space is chosen to be all the possible state transitions in a single stage of the trellis. We are interested in trelis waveform coders with rate 1 bitsample. This imposes a constraint on the encoder structure: from each node, there are two outgoing branches which correspond to values of 0 and 1 for the binary channel code. We also constrain the number of input branches going into each node: there are two incoming branches. This constraint is imposed in order to obtain a more symmetric structure so that the search space is minimized and the possibility of pathological trellis structures is certainly eliminated. The move set has been chosen to be just the flipping of two branches, so that the output of a move is again in the state space. The cost function is simply the minimum metric calculated by VA. The initial value of the control parameter is calculated as suggested by Johnson et al. [5]. Geometric improvement is used as the cooling schedule. The length of Metropolis loops are determined experimentally. As the source, a first order Gauss-Markov source with autocorrelation coefficient 0.9 is used. This source is chosen since it is a common model of real data and it is widely used in comparing data compression systems. For the design of codewords, we used the generalized Lloyd algorithm (GLA) [1].

In this work, GLA and SA are run together. For a given codebook, the trellis structure is optimized using SA, and for this structure, the codebook is modified using GLA. The process is stopped when the system reaches an equilibrium, with respect to the SA criteria.

\section{Results}

Trellis waveform coding systems of different constraint lengths were trained using a first order Gauss-Markov source, and were coded using SA and GLA. For constraint lengths of $K=2-8$, signal-to-quantization-noise ratios (SQNR) were computed. Then the system was tested using another first order Gauss-Markov source. In Table 1, the computed SQNR [dB] values are given (SA+GLA) together with the results of Stewart et al. (GLA) [1], and of Ayanoglu and Gray (PS) [6]. Results obtained using SA are better than those of [1]. This is expected since in [1] the trellis structure was fixed, not optimized. The results obtained via the predictive system [6] are better than the combined system, especiality for low constraint lengths. Again, this is expected since the predictive system has a higher system complexity. However, our results are sufficiently close to those of [6] for intermediate constraint lengths, so that the nonpredictive system once again becomes attractive. Alternatively, SA can be incorporated into the predictive system design with possibly better performance.

\section{References}

[1] L. C. Stewart, R. M. Gray, Y. Linde, "The Design of Trellis Waveform Coders," IEEE Trans. Comm., Vol. COM-30, pp. 702-711, April 1982.

[2] G. H. Freeman, J. W. Mark, I. F. Blake, "Trellis Source Codes Designed by Conjugate Gradient Optimization," IEEE Trans. Comm., Vol. COM36. pp. 1-12, January 1988.

[3] J. Foster, R. M. Gray, M. O. Dunham, "Finite-State Vector Quantization for Waveform Coding," IEEE Trans. Info. Theo., Vol. IT-31, pp. 348-359, May 1985.

[4] S. Kirkpatrick, C. D. Gelatt, M. P. Vecchi, "Optimization by Simulated Annealing," Science, Vol. 220, pp. 671-680, May 1983.

[5] D. S. Johnson, C. R. Aragon, L. A. McGeoch, C. Shevon, 'Optimization by Simulated Annealing: an Experimental Evaluation, Parts I and II,' Operations Research, Vol. 37, pp. 865-892, December 1989, and Vol. 39, pp. 378-406, June 1991.

[6] E. Ayanoglu, R. M. Gray, "The Design of Predictive Trellis Waveform Coders Using the Generalized Lloyd Algorithm," IEEE Trans. Comm., Vol. COM-34, pp. 1073-1081, November 1986.

\begin{tabular}{||c|c|c|c|c|c|c||}
\hline & \multicolumn{2}{|c|}{ SA+GLA } & \multicolumn{2}{c|}{ GLA } & \multicolumn{2}{c|}{ PS } \\
\hline$K$ & train & test & train & test & train & test \\
\hline 2 & 6.92 & 6.86 & 6.92 & 6.86 & 11.08 & 10.73 \\
3 & 9.81 & 9.45 & 8.77 & 8.59 & 11.53 & 11.18 \\
4 & 11.24 & 11.13 & 10.13 & 9.87 & 11.84 & 11.47 \\
5 & 11.90 & 11.77 & 11.05 & 10.67 & 12.18 & 11.83 \\
6 & 12.00 & 11.90 & 11.56 & 11.09 & 12.38 & 11.96 \\
7 & 12.29 & 11.98 & 11.87 & 11.70 & 12.52 & 12.52 \\
8 & 12.32 & 11.97 & 12.13 & 11.91 & 12.64 & 12.58 \\
\hline
\end{tabular}

Table 1: SQNR [dB] values, SA+GLA: Simulated Annealing and Generalized Lloyd Algorithm, GLA: Generalized Lloyd Algorithm only, PS: Predictive System. K: Constraint Length. 